\title{
A Mean-Value Inequality for Non-negative Solutions to the Linearized Monge-Ampère Equation
}

\author{
Liliana Forzani • Diego Maldonado
}

Received: 28 July 2008 / Accepted: 16 December 2008 / Published online: 13 January 2009

(C) Springer Science + Business Media B.V. 2009

\begin{abstract}
We prove a mean value inequality for non-negative solutions to $\mathcal{L}_{\varphi} u=0$ in any domain $\Omega \subset \mathbb{R}^{n}$, where $\mathcal{L}_{\varphi}$ is the Monge-Ampère operator linearized at a convex function $\varphi$, under minimal assumptions on the Monge-Ampère measure of $\varphi$. An application to the Harnack inequality for affine maximal hypersurfaces is included.
\end{abstract}

Keywords Linearized Monge-Ampère equation • Monge-Ampère measure

Mathematics Subject Classifications (2000) Primary 35J60 • 35D10 •

Secondary 26B25

\section{Introduction}

Let $u \in C^{2}\left(\mathbb{R}^{n}\right)$ and denote by $D^{2} u$ its Hessian matrix. If det $D^{2} u$ does not vanish, the Monge-Ampère operator $u \mapsto \operatorname{det} D^{2} u$ has the expansion

$$
\operatorname{det} D^{2}(u+t v)=\operatorname{det} D^{2} u+t \operatorname{trace}\left(\operatorname{det} D^{2} u\left(D^{2} u\right)^{-1} D^{2} v\right)+\cdots+t^{n} \operatorname{det} D^{2} v,
$$

L. Forzani

UNL-IMAL-CONICET, Santa Fe, Argentina

L. Forzani

School of Statistics, University of Minnesota, 313 Ford Hall, 224 Church Street,

Minneapolis, MN 55455, USA

e-mail: lforzani@math.unl.edu.ar

D. Maldonado $(\varangle)$

Department of Mathematics, Kansas State University, 138 Cardwell Hall,

Manhattan, KS 66506, USA

e-mail: dmaldona@math.ksu.edu 
for $t \in \mathbb{R}$ and $v \in C^{2}\left(\mathbb{R}^{n}\right)$. This expansion allows to define $\mathcal{L}_{u}$, the linearization of the Monge-Ampère operator at $u$, by

$$
\mathcal{L}_{u}(v)=\operatorname{trace}\left(A_{u} D^{2} v\right),
$$

where $A_{u}=\operatorname{det} D^{2} u\left(D^{2} u\right)^{-1}$. The linearized Monge-Ampère operator finds applications in diverse areas of Mathematics and Physics such as fluid mechanics [2], [10], real analysis [8], and differential geometry [21-23]. The case in which the linearization is done at a strictly convex function $\varphi: \mathbb{R}^{n} \rightarrow \mathbb{R}$ makes $\mathcal{L}_{\varphi}$ an elliptic, possibly degenerate, operator. Moreover, this case provides the measure theoretic and geometric elements to examine the properties of the solutions to $\mathcal{L}_{\varphi}(u)=0$. Indeed, following L. Caffarelli in [4], given a strictly convex, differentiable function $\varphi: \mathbb{R}^{n} \rightarrow \mathbb{R}, x \in \mathbb{R}^{n}$, and $r>0$, a section of $\varphi$ centered in $x$ at height $r$ is the open bounded convex set

$$
S_{\varphi}(x, r)=\left\{y \in \mathbb{R}^{n}: \varphi(y)<\varphi(x)+\langle\nabla \varphi(x), y-x\rangle+r\right\} .
$$

The Monge-Ampère measure $\mu_{\varphi}$ associated to $\varphi$ is defined by

$$
d \mu_{\varphi}(x)=\operatorname{det} D^{2} \varphi(x) d x .
$$

Notice that for the particular choice $\varphi_{2}(x):=|x|^{2} / 2$, we have that

$$
S_{\varphi_{2}}(x, r)=B\left(x, r^{1 / 2}\right), \quad x \in \mathbb{R}^{n}, r>0,
$$

(where $B\left(x, r^{1 / 2}\right)$ is the usual Euclidean ball centered at $x$ and radius $r^{1 / 2}$ ), $\mu_{\varphi_{2}}$ reduces to Lebesgue measure, and $\mathcal{L}_{\varphi_{2}}=\Delta$.

The paradigm in the study of properties of solutions $u$ to $\mathcal{L}_{\varphi} u=0$ is based on making the sections of $\varphi$ and the Monge-Ampère measure $\mu_{\varphi}$ play the roles that the usual Eucliden balls and Lebesgue measure play in the case of the Laplacian operator. Along these lines, some properties of the sections and Monge-Ampère measure, that emulate those of $\varphi_{2}$, are defined for a general strictly convex differentiable function $\varphi$.

Definition 1 Following Caffarelli and Gutiérrez in [6, 7] (see also [15, 16]) we say that $\mu_{\varphi}$ satisfies the (DP)-doubling property, and write $\mu \in(D P)$, if there exists a constant $C \geq 1$ such that

$$
\mu_{\varphi}\left(S_{\varphi}(x, r)\right) \leq C \mu_{\varphi}\left(S_{\varphi}(x, r / 2)\right), \quad x \in \mathbb{R}^{n}, r>0 .
$$

We say that $\mu_{\varphi}$ satisfies the (DC)-doubling property if there exist constants $B_{0} \geq 1$ and $0<\alpha<1$ such that for all sections $S_{\varphi}(x, r)$, we have

$$
\mu_{\varphi}\left(S_{\varphi}(x, r)\right) \leq B_{0} \mu_{\varphi}\left(\alpha S_{\varphi}(x, r)\right)
$$

where $\alpha S_{\varphi}(x, r)$ denotes $\alpha$-dilation of $S_{\varphi}(x, r)$ with respect to its center of mass $x^{*}$, that is,

$$
\alpha S_{\varphi}(x, r)=\left\{\alpha\left(y-x^{*}\right)+x^{*}: y \in S_{\varphi}(x, r)\right\},
$$

(centers of mass will be always considered with respect to Lebesgue measure). 
We say that $\mu_{\varphi}$ verifies the $\left(\mu_{\infty}\right)$-condition, and write $\mu_{\varphi} \in\left(\mu_{\infty}\right)$ or $\mu_{\varphi} \in\left(\mu_{\infty}\left(\mathbb{R}^{n}\right)\right)$, if given $\varepsilon \in(0,1)$ there exists $\delta \in(0,1)$ such that for every section $S=S_{\varphi}(x, r)$ and every measurable set $E \subset S$,

$$
\frac{|E|}{|S|}<\delta \Rightarrow \frac{\mu_{\varphi}(E)}{\mu_{\varphi}(S)}<\varepsilon,
$$

(if $U \subset \mathbb{R}^{k}$, for some $k \in \mathbb{N}$, then $|U|$ denotes its $k$-dimensional Lebesgue measure). Finally, we say that the sections of $\varphi$ verify the engulfing property if there exists a constant $K \geq 1$ such that whenever $x \in S_{\varphi}(y, r)$ then

$$
S_{\varphi}(y, r) \subset S_{\varphi}(x, K r) .
$$

In this case we write $\varphi \in \operatorname{Eng}(n, K)$.

The study of the regularity properties of non-negative solutions to $\mathcal{L}_{\varphi} u=0$ has been pioneered by L. Caffarelli and C. Gutiérrez in [7]. Among their results, we first single out

Theorem 2 (Harnack's inequality. Theorem 5 in [7]). Suppose $\mu_{\varphi} \in\left(\mu_{\infty}\right)$, then there exist constants $\beta>1>\tau>0$, depending only on the $\left(\mu_{\infty}\right)$ condition and dimension $n$, such that if $u$ is any non-negative solution of $\mathcal{L}_{\varphi} u=0$ in the section $S_{\varphi}(z, t)$ then

$$
\sup _{S_{\varphi}(z, \tau t)} u \leq \beta \inf _{S_{\varphi}(z, \tau t)} u .
$$

In this article we first show that the (DC)-doubling condition is significantly weaker than the $\left(\mu_{\infty}\right)$ condition in every dimension. Then, we explore some properties of non-negative solutions $u$ to $\mathcal{L}_{\varphi}(u)=0$ merely under the (DC)-doubling assumption. Our main result is the following mean value inequality

Theorem 3 Assume that $\mu_{\varphi}$ verifies the (DC)-doubling property. There exists a constant $B_{1}>0$, depending only on $B_{0}$ and $\alpha$ in $E q .1 .1$ and $n$, such that for every open subset $\Omega \subset \mathbb{R}^{n}$ and all $u \geq 0$ in $\Omega$ satisfying $\mathcal{L}_{\varphi}(u)=0$ in $\Omega$ we have

$$
\sup _{S_{\varphi}(x, r / 8)} u \leq \frac{B_{1}}{\mu_{\varphi}\left(S_{\varphi}(x, r)\right)} \int_{S_{\varphi}(x, r)} u d \mu_{\varphi},
$$

for every section $S_{\varphi}(x, r)$ with $S_{\varphi}(x, 2 r) \subset \subset \Omega$.

As an application of Theorem 3, in Section 7 we prove Harnack's inequality for affine maximal hypersurfaces that verify the (DC)-doubling property.

Remark 4 Given a bounded open subset $\Omega \subset \mathbb{R}^{n}$ and $\varphi \in C^{2}\left(\mathbb{R}^{n}\right)$ with $D^{2} \varphi>0$, the matrix $D^{2} \varphi$ will always be strictly elliptic in $\Omega$, with constants depending on the maximum and minimum eigenvalues of $D^{2} \varphi$ over $\bar{\Omega}$. Elliptic regularity then implies that solutions to $\mathcal{L}_{\varphi} u=0$ in $\Omega$ will enjoy properties such as Harnack inequalities, Hölder continuity, $L^{p}$-estimates, etc. However, the strength of theorems such as Theorem 2 and Theorem 3 resides in the fact that the constants involved are independent of $\Omega$ and, moreover, they depend on $D^{2} \varphi$ only through its determinant. 
Throughout this article, the equation $\mathcal{L}_{\varphi} u=0$ can be interpreted to be satisfied in the classical sense. However, all our estimates will depend only upon dimension and the doubling constant in Eq. 1.1; in particular, they are independent of any smoothness assumption on the solutions.

\section{Real Analysis Associated to the Monge-Ampère Equation}

The relevance of the (DC)-doubling condition in the regularity theory for the Monge-Ampère equation was first demonstrated by the celebrated $C^{1, \alpha}$-regularity theorem for solutions to Monge-Ampère equations due to L. Caffarelli [4]. Since then, this doubling condition has been intensively studied and related to other measure theoretic and geometric properties, see, for instance, [4-7, 12, 13, 15], and [16]. In fact, the following equivalences (see Theorem 8 in [12] and Theorem 4 in [13]) hold true

Theorem 5 Let $\varphi: \mathbb{R}^{n} \rightarrow \mathbb{R}$ be a differentiable strictly convex function. Then the following are equivalent

(i) the measure $\mu_{\varphi}$ satisfies the (DC)-doubling property,

(ii) the Monge-Ampère measure $\mu_{\varphi}$ satisfies

$$
c r^{n} \leq\left|S_{\varphi}(x, r)\right| \mu_{\varphi}\left(S_{\varphi}(x, r)\right) \leq C r^{n},
$$

for all sections $S_{\varphi}(x, r)$ and some positive constants $c, C$;

(iii) the sections of $\varphi$ satisfy the engulfing property.

The statements are quantitative in the sense that the constants involved in each property depend only on each other and not on $\varphi$

The (DC)-doubling property provides a measure theoretic and geometric structure rich enough to do real analysis. Indeed, if we set

$$
\rho_{\varphi}(x, y):=\inf \left\{r>0: y \in S_{\varphi}(x, r), x \in S_{\varphi}(y, r)\right\}, \quad x, y \in \mathbb{R}^{n},
$$

and if $\mu_{\varphi}$ verifies Eq. 1.1, then $\rho_{\varphi}$ becomes a quasi-distance on $\mathbb{R}^{n}$ (with quasi-triangle constant depending only on $B_{0}, \alpha$, and $n$ ) whose balls are topologically equivalent to the sections of $\varphi$. That is, there exist positive constants $0<\delta_{1}<1<\delta_{2}$, depending only on $B_{0}$ and $\alpha$ in Eq. 1.1, such that

$$
S_{\varphi}\left(x, \delta_{1} r\right) \subset B_{\rho_{\varphi}}(x, r) \subset S_{\varphi}\left(x, \delta_{2} r\right), \quad x \in \mathbb{R}^{n}, r>0,
$$

where $B_{\rho_{\varphi}}(x, r)=\left\{y \in \mathbb{R}^{n}: \rho_{\varphi}(x, y)<r\right\}$. This result was proved by H. Aimar, L. Forzani, and R. Toledano in [1] under the assumption of several geometric conditions on the sections of $\varphi$, which, later on, turned out to be equivalent to the (DC)-doubling condition, (see Theorem 8 in [12]). Conversely, if Eq. 2.3 holds for some quasidistance $\rho$ instead of $\rho_{\varphi}$, then its quasi-triangle inequality implies that the sections of $\varphi$ verify the engulfing property. Hence, the (DC)-doubling condition for $\mu_{\varphi}$ is equivalent to the existence of a quasi-distance in $\mathbb{R}^{n}$ whose balls are topologically equivalent to the sections of $\varphi$.

The (DC)-doubling property implies the (DP)-doubling property, with $C=$ $C\left(\alpha, B_{0}, n\right)$ (however, the converse is not true, see [15], p. 51). Thus, in the pres- 
ence of the (DC)-doubling property, the triple $\left(\mathbb{R}^{n}, \mu_{\varphi}, \rho_{\varphi}\right)$ becomes a space of homogeneous type. Note that the mean value inequality in Theorem 3 is related to $\left(\mathbb{R}^{n}, \mu_{\varphi}, \rho_{\varphi}\right)$. For more on the real analysis related to $\varphi$, see the fundamental work [6].

\section{The (DC)-Doubling Condition vs. the $\left(\mu_{\infty}\right)$ Condition}

Given a strictly convex, differentiable function $\varphi: \mathbb{R} \rightarrow \mathbb{R}$, every open interval in the real line can be realized as a section of $\varphi$ (just by the mean value theorem). Hence, the (DC)-doubling property becomes the usual doubling property for the measure $\varphi^{\prime \prime}$. Similarly, the condition $\left(\mu_{\infty}\right)$ becomes the $A_{\infty}$ condition for Muckenhoupt weights. It is therefore clear that, at least in dimension 1, the (DC) doubling condition is considerably weaker than the $\left(\mu_{\infty}\right)$ condition (the gap being exactly the one between doubling measures and Muchenhoupt weights). The following lemma will be useful to produce examples for the (DC) and $\left(\mu_{\infty}\right)$ conditions when $n>1$.

Lemma 6 Fix $m \in \mathbb{N}$. For each $j=1, \ldots, m$ consider a strictly convex, differentiable function $\varphi_{j}: \mathbb{R}^{n_{j}} \rightarrow \mathbb{R}$. Set $n=\sum_{j=1}^{m} n_{j}$ and define $\varphi: \mathbb{R}^{n} \rightarrow \mathbb{R}$ as the tensor sum of the $\varphi_{j}$ 's. That is,

$$
\varphi(x)=\sum_{j=1}^{m} \varphi_{j}\left(x_{j}\right), \quad x=\left(x_{1}, x_{2}, \ldots, x_{m}\right) \in \mathbb{R}^{n}, x_{j} \in \mathbb{R}^{n_{j}}, j=1, \ldots, m .
$$

Then,

$$
S_{\varphi}(x, t) \subset \prod_{j=1}^{m} S_{\varphi_{j}}\left(x_{j}, t\right) \subset S_{\varphi}(x, m t),
$$

for all $x=\left(x_{1}, x_{2}, \ldots, x_{m}\right) \in \mathbb{R}^{n}$ and $t>0$. In particular,

(i) if $\varphi_{j} \in \operatorname{Eng}\left(n_{j}, K_{j}\right)$ then $\varphi \in \operatorname{Eng}(n, m K)$, where

$$
K=\max \left\{K_{j}: j=1, \ldots, m\right\} .
$$

Conversely, if $\varphi \in \operatorname{Eng}\left(n, K^{\prime}\right)$, for some $K^{\prime}>1$, then $\varphi_{j} \in \operatorname{Eng}\left(n_{j}, K^{\prime}\right)$, $j=1, \ldots, m$;

(ii) if $\mu_{j}:=\operatorname{det} D^{2} \varphi_{j} \in\left(\mu_{\infty}\left(\mathbb{R}^{n_{j}}\right)\right), j=1, \ldots, m$, then $\mu_{\varphi} \in\left(\mu_{\infty}\left(\mathbb{R}^{n}\right)\right)$. Conversely, if $\mu_{\varphi} \in\left(\mu_{\infty}\left(\mathbb{R}^{n}\right)\right)$, then $\mu_{j} \in\left(\mu_{\infty}\left(\mathbb{R}^{n_{j}}\right)\right), j=1, \ldots, m$.

Proof By definition of the sections of $\varphi$, we have that $y \in S_{\varphi}(x, t)$ if and only if

$$
\varphi(y)<\varphi(x)+\langle\nabla \varphi(x), y-x\rangle+t,
$$

which means $\sum_{j=1}^{m}\left(\varphi_{j}\left(y_{j}\right)-\varphi_{j}\left(x_{j}\right)-\left\langle\nabla_{x_{j}} \varphi_{j}\left(x_{j}\right), y_{j}-x_{j}\right\rangle\right)<t$. Since convexity implies that each of the summands is non-negative, we have

$$
\varphi_{j}\left(y_{j}\right)-\varphi_{j}\left(x_{j}\right)-\left\langle\nabla_{x_{j}} \varphi_{j}\left(x_{j}\right), y_{j}-x_{j}\right\rangle<t, \quad j=1, \ldots, m .
$$

That is, $y_{j} \in S_{\varphi_{j}}\left(x_{j}, t\right), j=1, \ldots, m$. This proves the first inclusion. The second inclusion follows by using that $y_{j} \in S_{\varphi_{j}}\left(x_{j}, t\right)$ if and only if Eq. 3.5 and by adding together the inequalities in Eq. 3.5 for $j=1, \ldots, m$. In order to prove (i), assume 
that $\varphi_{j} \in \operatorname{Eng}\left(n_{j}, K_{j}\right)$. If $y \in S_{\varphi}(x, t)$, then by Eq. 3.4, $y_{j} \in S_{\varphi_{j}}\left(x_{j}, t\right), j=1, \ldots, m$, and by the engulfing property, $x_{j} \in S_{\varphi_{j}}\left(y_{j}, K_{j} t\right) \subset S_{\varphi_{j}}\left(y_{j}, K t\right), j=1, \ldots, m$. Therefore, by Eq. 3.4, $x \in S_{\varphi}(y, m K t)$. Thus, $\varphi \in \operatorname{Eng}(n, m K)$. Conversely, if $\varphi \in \operatorname{Eng}\left(n, K^{\prime}\right)$, fix $j \in\{1, \ldots, m\}$ and for each $x_{j} \in \mathbb{R}^{n_{j}}$ set $\tilde{x}_{j}:=\left(0, \ldots, x_{j}, \ldots, 0\right)$, so that $y_{j} \in$ $S_{\varphi_{j}}\left(x_{j}, t\right)$ if and only if $\tilde{y}_{j} \in S_{\varphi}\left(\tilde{x}_{j}, t\right)$. Therefore, $x_{j} \in S_{\varphi_{j}}\left(n_{j}, K^{\prime}\right)$ and, consequently, $\varphi_{j} \in \operatorname{Eng}\left(n_{j}, K^{\prime}\right), j=1, \ldots, m$. In order to prove (ii), we recall that (as indicated in [7], p. 465) $\mu_{\varphi} \in \mu_{\infty}\left(\mathbb{R}^{n}\right)$ if and only if there exist constants $1<C, r<\infty$ such that for all the sections $S=S_{\varphi}(x, t)$ the following reverse Hölder inequality holds true,

$$
\left(\frac{1}{|S|} \int_{S} \mu_{\varphi}(y)^{r} d y\right)^{1 / r} \leq C \frac{1}{|S|} \int_{S} \mu_{\varphi}(y) d y .
$$

If $\mu_{j} \in\left(\mu_{\infty}\left(\mathbb{R}^{n_{j}}\right)\right), \mu_{j}$ satisfies Eq. 3.6 with some constants $1<C_{j}, r_{j}<\infty$. Set $r=$ $\min \left\{r_{j}: j=1, \ldots, m\right\}>1$ and $C=\prod_{j=1}^{m} C_{j}^{1 / r}>1$. By Lemma 5.2 in [7], for any $k \in$ $\mathbb{N}, k$-dimensional Lebesgue measure is doubling with respect to the parameter $t$ of the sections of every strictly convex, differentiable function $\psi$ defined on $\mathbb{R}^{k}$. Moreover,

$$
\left|S_{\psi}(x, t)\right| \leq 2^{k}\left|S_{\psi}(x, t / 2)\right|, \quad x \in \mathbb{R}^{k}, t>0 .
$$

Given a section $S_{\varphi}(x, t)$, we now use Eqs. 3.4 and 3.7 to write

$$
\begin{aligned}
\left(\frac{1}{\left|S_{\varphi}(x, t)\right|} \int_{S_{\varphi}(x, t)} \mu(y)^{r} d y\right)^{1 / r} & =\left(\frac{1}{\left|S_{\varphi}(x, t)\right|} \int_{S_{\varphi}(x, t)} \prod_{j=1}^{m} \mu_{j}\left(y_{j}\right)^{r} d y\right)^{1 / r} \\
& \leq \prod_{j=1}^{m}\left(\frac{1}{\left|S_{\varphi_{j}}\left(x_{j}, t / m\right)\right|} \int_{S_{\varphi_{j}}\left(x_{j}, t\right)} \mu_{j}\left(y_{j}\right)^{r} d y_{j}\right)^{1 / r} \\
& \leq \prod_{j=1}^{m}\left(\frac{C_{n_{j}, m}}{\left|S_{\varphi_{j}}\left(x_{j}, t\right)\right|} \int_{S_{\varphi_{j}}\left(x_{j}, t\right)} \mu_{j}\left(y_{j}\right)^{r} d y_{j}\right)^{1 / r} \\
& \leq C \prod_{j=1}^{m} \frac{C_{n_{j}, m}}{\left|S_{\varphi_{j}}\left(x_{j}, t\right)\right|} \int_{S_{\varphi_{j}}\left(x_{j}, t\right)} \mu_{j}\left(y_{j}\right) d y_{j} \\
& \leq C\left(\prod_{j=1}^{m} C_{n_{j}, m}\right) \frac{1}{\left|S_{\varphi}(x, t)\right|} \int_{S_{\varphi}(x, t)} \mu_{\varphi}(y) d y .
\end{aligned}
$$

To prove the converse in (ii), suppose that $\mu_{\varphi} \in\left(\mu_{\infty}\left(\mathbb{R}^{n}\right)\right)$ satisfies Eq. 3.6 with constants $1<C, r<\infty$. By Eqs. 3.4, 3.6, and 3.7 there exists a constant $C^{*}$, depending only on $C, r, m$, and $n$ such that

$$
1 \leq \prod_{j=1}^{m} \frac{\left(\frac{1}{\left|S_{\varphi_{j}}(x, t)\right|} \int_{S_{\varphi_{j}}\left(x_{j}, t\right)} \mu_{j}\left(y_{j}\right)^{r} d y_{j}\right)^{1 / r}}{\frac{1}{\left|S_{\varphi_{j}}\left(x_{j}, t\right)\right|} \int_{S_{\varphi_{j}}\left(x_{j}, t\right)} \mu_{j}\left(y_{j}\right) d y_{j}} \leq C^{*},
$$

since each factor in Eq. 3.8 is greater than or equal to one, it follows that $\mu_{j}$ verifies a reverse Hölder inequality with constants $1<C^{*}, r<\infty$. 
Examples of doubling measures which are singular with respect to Lebesgue measure, as well as examples of continuous (and even Lipschitz continuous) doubling weights which are not in $A_{\infty}$, can be constructed on the real line (see, for instance, [20, p. 40], [9, Examples 5.2 and 5.3], and [24, Theorem 4]). Integrating such measures twice produces convex functions defined on the real line whose sections satisfy the engulfing property. By Theorem 5 and Lemma 6, tensor sums of these onedimensional convex functions provide higher dimensional examples of strictly convex functions whose Monge-Ampère measures verify the (DC)-doubling condition, but not the $\left(\mu_{\infty}\right)$ condition.

\section{The Normalization Technique}

Let $T: \mathbb{R}^{n} \rightarrow \mathbb{R}^{n}$ be an invertible affine transformation and, for $\varphi$ as above, define $\psi(y)=\varphi\left(T^{-1} y\right)$. Hence, $T\left(S_{\varphi}(x, r)\right)=S_{\psi}(T x, r)$ and $T\left(\alpha S_{\varphi}(x, r)\right)=\alpha T\left(S_{\varphi}(x, r)\right)$. Thus, $\mu_{\psi}$ verifies the (DC)-doubling property (Eq. 1.1) with the same constants $B_{0}$ and $\alpha$ as $\varphi$ does. Moreover, if $v$ is defined as $v(y)=u\left(T^{-1} y\right)$, then $\mathcal{L}_{\varphi} u=0$ on $S_{\varphi}(x, r)$ if and only if $\mathcal{L}_{\psi} v=0$ on $S_{\psi}(T x, r)$. In particular, quantities such as

$$
\inf _{S_{\varphi}(x, r)} u, \quad \sup _{S_{\varphi}(x, r)} u \text {, and } \frac{1}{\mu_{\varphi}\left(S_{\varphi}(x, r)\right)} \int_{S_{\varphi}(x, r)} u d \mu_{\varphi},
$$

are preserved under the affine transformations that take $\varphi$ into $\psi$ and $u$ into $v$. See [7], Section 1, for more details. For the following lemma and other basic results on the Monge-Ampère equation, the reader is referred to [15].

Lemma 7 (John's Lemma) Let $\Omega \subset \mathbb{R}^{n}$ be a bounded and convex set with non-empty interior. Consider all the ellipsoids that contain $\Omega$ and that are centered at the center of mass of $\Omega$. Let $E$ be the ellipsoid of minimum volume. Then, there is a constant $\alpha_{n}>0$ depending only on $n$ such that

$$
\alpha_{n} E \subset \Omega \subset E
$$

A convex set $\Omega \subset \mathbb{R}^{n}$ is said to be normalized if its center of mass is 0 and $B\left(0, \alpha_{n}\right) \subset \Omega \subset B(0,1)$. Given any bounded convex subset $S$ with non-empty interior, let $E_{S}$ be the ellipsoid given by John's Lemma applied to $S$; and $T: \mathbb{R}^{n} \rightarrow \mathbb{R}^{n}$ be an affine transformation with $T\left(E_{S}\right)=B(0,1)$, we then have

$$
B\left(0, \alpha_{n}\right) \subset T(S) \subset B(0,1) .
$$

We call $T(S)$ the normalization of $S$ (through $T$ ). From now on, we fix the notation

$$
L_{\varphi} u:=\operatorname{trace}\left(\left(D^{2} \varphi\right)^{-1} D^{2} u\right),
$$

so that

$$
\mathcal{L}_{\varphi} u=\operatorname{det} D^{2} \varphi L_{\varphi} u
$$

Lemma 8 If $u$ is a solution to $L_{\varphi} u=f$ in $S_{\varphi}(x, r)$ and $u=0$ on $\partial S_{\varphi}(x, r)$ then

$$
\sup _{S_{\varphi}(x, r)}|u| \leq C_{n}\left|S_{\varphi}(x, r)\right|^{1 / n}\left(\int_{S_{\varphi}(x, r)}|f|^{n} d \mu_{\varphi}\right)^{1 / n} .
$$


Proof Let $T$ be an affine transformation that normalizes $S_{\varphi}(x, r)$. Define $v(y)=$ $u\left(T^{-1} y\right)$ and $\psi(y)=\varphi\left(T^{-1} y\right)$. Thus, $\mathcal{L}_{\psi} v(y)=\mathcal{L}_{\varphi} u\left(T^{-1} y\right)$ for $y \in S_{\psi}(T x, r)$ and $B\left(0, \alpha_{n}\right) \subset S_{\psi}(T x, r) \subset B(0,1)$. Now, by Aleksandrov's maximum principle (see [14], p. 222), we have

$$
\left.\sup _{S_{\psi}(T x, r)}|v| \leq c_{n} \operatorname{diam}\left(S_{\psi}(T x, r)\right) \| \mathcal{L}_{\psi} v / d^{*}\right) \|_{L^{n}\left(S_{\psi}(T x, r)\right)},
$$

where

$$
d^{*}(y)=\left(\operatorname{det}\left(D^{2} \psi\right)^{-1}(y)\right)^{1 / n}=\left(\operatorname{det}\left(D^{2} \varphi\right)^{-1}\left(T^{-1} y\right)(\operatorname{det} T)^{2}\right)^{1 / n}
$$

and $c_{n}$ is a dimensional constant. We now use that $S_{\psi}(T x, r)$ is normalized to produce a dimensional constant $\tilde{c}_{n}$ such that

$$
\operatorname{diam}\left(S_{\psi}(T x, r)\right) \leq \tilde{c}_{n}\left|S_{\psi}(T x, r)\right|^{1 / n} .
$$

Since $\left|S_{\psi}(T x, r)\right|=|\operatorname{det} T|\left|S_{\varphi}(x, r)\right|$, it follows that

$$
\left.\sup _{S_{\psi}(T x, r)}|v| \leq c_{n} \tilde{c_{n}}|\operatorname{det} T|^{1 / n}\left|S_{\varphi}(x, r)\right|^{1 / n} \| \mathcal{L}_{\psi} v / d^{*}\right) \|_{L^{n}\left(S_{\psi}(T x, r)\right)} .
$$

The lemma follows just by changing variables $y \mapsto T^{-1} y$ back in Eq. 4.9. The constant $C_{n}$ turns out to be $c_{n} \tilde{c}_{n}$, depending only on the dimension $n$.

\section{A Weak Reverse Hölder Inequality for Non-negative Solutions}

For $\varphi \in C^{2}(\Omega)$, it can be checked (by plain differentiation) that the operator $\mathcal{L}_{\varphi}$ enjoys the following null-Lagrangian property

$$
\mathcal{L}_{\varphi} v=\operatorname{trace}\left(A_{\varphi} D^{2} v\right)=\operatorname{div}\left(A_{\varphi} \nabla v\right) .
$$

Hence, if $v$ is a solution to $\mathcal{L}_{\varphi} v=0$ in $\Omega$, integrating by parts we get

$$
\int_{\Omega} v(y) L_{\varphi} w(y) d \mu_{\varphi}(y)=0,
$$

for all $w \in C_{0}^{1}(\Omega)$. In this section we prove

Theorem 9 Assume that $\mu_{\varphi}$ verifies the (DC)-doubling property. There exists a constant $B_{2}>0$, depending only on $B_{0}$ and $\alpha$ in Eq. 1.1 and $n$, such that for every open subset $\Omega \subset \mathbb{R}^{n}$ and all $v \geq 0$ in $\Omega$ satisfying $\mathcal{L}_{\varphi}(v)=0$ in $\Omega$ we have

$$
\left(\frac{1}{\mu_{\varphi}\left(S_{\varphi}(x, r)\right)} \int_{S_{\varphi}(x, r)} v^{\frac{n}{n-1}} d \mu_{\varphi}\right)^{\frac{n-1}{n}} \leq \frac{B_{2}}{\mu_{\varphi}\left(S_{\varphi}(x, 2 r)\right)} \int_{S_{\varphi}(x, 2 r)} v d \mu_{\varphi},
$$

for every section $S_{\varphi}(x, r)$ with $S_{\varphi}(x, 2 r) \subset \subset \Omega$.

Proof Without loss of generality we can assume that $\varphi(0)=0$ and $\nabla \varphi(0)=0$, since subtracting a hyperplane from $\varphi$ does not change the operator $\mathcal{L}_{\varphi}$ or the measure $\mu_{\varphi}$. In particular, we can assume that $\varphi \geq 0$. 
To begin with, let us consider first the section $S_{\varphi}(0, r)$. We can bound the $L_{\mu_{\varphi}}^{\frac{n}{n-1}}-$ norm of $v$ over $S_{\varphi}(0, r)$ by estimating

$$
\sup \left\{\int_{S_{\varphi}(0, r)} v(y) f(y) d \mu_{\varphi}(y): f \in \mathcal{A}\right\},
$$

where

$$
\mathcal{A}:=\left\{f \in C_{c}^{\infty}\left(S_{\varphi}(0, r)\right): f \geq 0,\|f\|_{L_{\mu_{\varphi}}^{n}\left(S_{\varphi}(0, r)\right)} \leq 1\right\} .
$$

This approach to proving reverse Hölder inequalities for non-negative solutions to elliptic operators can be traced back, at least, to the work of E. Fabes and W. Stroock on uniformly elliptic equations in [11]. We will see how Theorem 5 allows to implement this technique in the current degenerate case.

Given $f \in \mathcal{A}$, we extend it as zero outside of $S_{\varphi}(0, r)$ and consider the $C^{2}$ function $Z_{r}(y)$ satisfying

$$
\begin{cases}L_{\varphi}\left(Z_{r}\right)=f & \text { in } S_{\varphi}(0,2 r) \\ Z_{r}=0 & \text { on } \partial S_{\varphi}(0,2 r) .\end{cases}
$$

(Recall that we have fixed the notation $\mathcal{L}_{\varphi}=\operatorname{det} D^{2} \varphi L_{\varphi}$ ). A simple calculation yields

$$
L_{\varphi}\left((2 r-\varphi) Z_{r}\right)=(2 r-\varphi) L_{\varphi} Z_{r}+2\left\langle\nabla(2 r-\varphi),\left(D^{2} \varphi\right)^{-1} \nabla Z_{r}\right\rangle-Z_{r} n .
$$

For $y \in S_{\varphi}(0, r)$ we have $2 r-\varphi(y) \geq r$, and by Aleksandrov's maximum principle, $Z_{r} \leq 0$ in $S_{\varphi}(0,2 r)$. Thus,

$$
\begin{aligned}
& \int_{S_{\varphi}(0, r)} v(y) f(y) d \mu_{\varphi}(y) \\
& \leq \frac{1}{r} \int_{S_{\varphi}(0, r)} v(y)(2 r-\varphi(y)) L_{\varphi} Z_{r}(y) d \mu_{\varphi}(y) \\
& =\frac{1}{r} \int_{S_{\varphi}(0,2 r)} v(y)(2 r-\varphi(y)) L_{\varphi} Z_{r}(y) d \mu_{\varphi}(y) \\
& =\frac{1}{r} \int_{S_{\varphi}(0,2 r)} v(y) L_{\varphi}\left((2 r-\varphi) Z_{r}\right)(y) d \mu_{\varphi}(y) \\
& -\frac{1}{r} \int_{S_{\varphi}(0,2 r)} v(y) Z_{r}(y) L_{\varphi}(2 r-\varphi)(y) d \mu_{\varphi}(y) \\
& -\frac{2}{r} \int_{S_{\varphi}(0,2 r)} v(y)\left\langle\nabla(2 r-\varphi)(y),\left(D^{2} \varphi\right)^{-1}(y) \nabla Z_{r}(y)\right\rangle d \mu_{\varphi}(y) \\
& =: I_{1}+I_{2}+I_{3},
\end{aligned}
$$

where we used Eq. 5.13. If we set $w=(2 r-\varphi) Z_{r}$, we have $w=0$ and $\nabla w=0$ on $\partial S_{\varphi}(0,2 r)$. Thus, $w$ (extended as 0 outside $S_{\varphi}(0,2 r)$ ) can be taken as a test function in Eq. 5.10. Consequently, $I_{1}=0$. Now, by Lemma 8, for every $y \in S_{\varphi}(0,2 r)$ we have

$$
\left|Z_{r}(y)\right| \leq C_{n}\left|S_{\varphi}(0,2 r)\right|^{1 / n}
$$


Therefore,

$$
\begin{aligned}
\left|I_{2}\right| & \leq \int_{S_{\varphi}(0,2 r)} \frac{v(y)}{r}\left|Z_{r}(y)\right|\left|L_{\varphi}(2 r-\varphi)(y)\right| d \mu_{\varphi}(y) \\
& \leq \int_{S_{\varphi}(0,2 r)} \frac{n C_{n} v(y)}{r}\left|S_{\varphi}(0,2 r)\right|^{1 / n} d \mu_{\varphi}(y) \\
& \leq \frac{n C_{n} \tilde{C}}{\left(\mu_{\varphi}\left(S_{\varphi}(0,2 r)\right)^{1 / n}\right.} \int_{S_{\varphi}(0,2 r)} v(y) d \mu_{\varphi}(y),
\end{aligned}
$$

where we used Theorem 5, part (ii), so that

$$
\mu_{\varphi}\left(S_{\varphi}(0,2 r)\right)\left|S_{\varphi}(0,2 r)\right| \leq(\tilde{C} r)^{n},
$$

for some $\tilde{C}=\tilde{C}\left(B_{0}, \alpha, n\right)$. In order to find a bound for $I_{3}$, we proceed as follows. Since $\left(D^{2} \varphi\right)>0$, for any $\varepsilon>0$ we get

$$
\begin{aligned}
\left|\left\langle\nabla(2 r-\varphi),\left(D^{2} \varphi\right)^{-1} \nabla Z_{r}\right\rangle\right| \leq & \left(\left\langle\nabla(2 r-\varphi),\left(D^{2} \varphi\right)^{-1} \nabla(2 r-\varphi)\right\rangle\right)^{1 / 2} \\
& \times\left(\left\langle\nabla Z_{r},\left(D^{2} \varphi\right)^{-1} \nabla Z_{r}\right\rangle\right)^{1 / 2} \\
\leq & \frac{\left|S_{\varphi}(0, r)\right|^{1 / n}}{2 \varepsilon r}\left(\left\langle\nabla(2 r-\varphi),\left(D^{2} \varphi\right)^{-1} \nabla(2 r-\varphi)\right\rangle\right) \\
& +\frac{\varepsilon r}{2\left|S_{\varphi}(0, r)\right|^{1 / n}}\left(\left\langle\nabla Z_{r},\left(D^{2} \varphi\right)^{-1} \nabla Z_{r}\right\rangle\right) \\
= & \frac{\left|S_{\varphi}(0, r)\right|^{1 / n}}{4 \varepsilon r}\left(L_{\varphi}(2 r-\varphi)^{2}-2(2 r-\varphi) L_{\varphi}(2 r-\varphi)\right) \\
& +\frac{\varepsilon r}{4\left|S_{\varphi}(0, r)\right|^{1 / n}}\left(L_{\varphi} Z_{r}^{2}-2 Z_{r} L_{\varphi} Z_{r}\right) .
\end{aligned}
$$

Next, we use $Z_{r}^{2}$ and $(2 r-\varphi)^{2}$ (both extended as 0 outside $S_{\varphi}(0,2 r)$ ) as test functions in Eq. 5.10, inequality 5.14, and (ii) in Theorem 5 to estimate

$$
\begin{aligned}
\left|I_{3}\right| \leq & \frac{\left|S_{\varphi}(0,2 r)\right|^{1 / n}}{\varepsilon r^{2}} \int_{S_{\varphi}(0,2 r)}(2 r-\varphi) v\left|L_{\varphi}(2 r-\varphi)\right| d \mu_{\varphi} \\
& +\frac{\varepsilon}{\left|S_{\varphi}(0,2 r)\right|^{1 / n}} \int_{S_{\varphi}(0,2 r)} v\left|Z_{r} L_{\varphi} Z_{r}\right| d \mu_{\varphi} \\
\leq & \frac{2\left|S_{\varphi}(0,2 r)\right|^{1 / n}}{\varepsilon r} \int_{S_{\varphi}(0,2 r)} v\left|L_{\varphi}(2 r-\varphi)\right| d \mu_{\varphi}+\varepsilon C_{n} \int_{S_{\varphi}(0,2 r)} v\left|L_{\varphi} Z_{r}\right| d \mu_{\varphi} \\
= & \frac{2 n\left|S_{\varphi}(0,2 r)\right|^{1 / n}}{\varepsilon r} \int_{S_{\varphi}(0,2 r)} v d \mu+\varepsilon C_{n} \int_{S_{\varphi}(0,2 r)} v f d \mu_{\varphi} \\
\leq & \frac{2 n \tilde{C}}{\varepsilon \mu_{\varphi}\left(S_{\varphi}(0,2 r)\right)^{1 / n}} \int_{S_{\varphi}(0,2 r)} v d \mu_{\varphi}+\varepsilon C_{n} \int_{S_{\varphi}(0,2 r)} v f d \mu_{\varphi} \\
= & \frac{2 n \tilde{C}}{\varepsilon \mu_{\varphi}\left(S_{\varphi}(0,2 r)\right)^{1 / n}} \int_{S_{\varphi}(0,2 r)} v d \mu_{\varphi}+\varepsilon C_{n} \int_{S_{\varphi}(0, r)} v f d \mu_{\varphi} .
\end{aligned}
$$


Hence,

$$
\int_{S_{\varphi}(0, r)} v f d \mu_{\varphi} \leq \frac{\left(n C_{n} \tilde{C}+\frac{2 n \tilde{C}}{\varepsilon}\right)}{\mu\left(S_{\varphi}(0,2 r)\right)^{1 / n}} \int_{S_{\varphi}(0,2 r)} v d \mu_{\varphi}+\varepsilon C_{n} \int_{S_{\varphi}(0, r)} v f d \mu .
$$

Choosing $\varepsilon<1 / C_{n}$, depending only on $n$, yields

$$
\int_{S_{\varphi}(0, r)} v f d \mu \leq\left(\frac{n C_{n} \tilde{C}+\frac{2 n \tilde{C}}{\varepsilon}}{1-\varepsilon C_{n}}\right) \frac{1}{\mu_{\varphi}\left(S_{\varphi}(0,2 r)\right)^{1 / n}} \int_{S_{\varphi}(0,2 r)} v d \mu_{\varphi} .
$$

Therefore, the supremum in Eq. 5.12 can be bounded by

$$
\frac{\left(n C_{n} \tilde{C} \varepsilon+2 n \tilde{C}\right)}{\varepsilon\left(1-\varepsilon C_{n}\right) \mu_{\varphi}\left(S_{\varphi}(0,2 r)\right)^{1 / n}} \int_{S_{\varphi}(0,2 r)} v d \mu_{\varphi}
$$

and

$$
\left(\frac{1}{\mu_{\varphi}\left(S_{\varphi}(0, r)\right)} \int_{S_{\varphi}(0, r)} v^{\frac{n}{n-1}} d \mu_{\varphi}\right)^{\frac{n-1}{n}} \leq \frac{B_{2}}{\mu_{\varphi}\left(S_{\varphi}(0,2 r)\right)} \int_{S_{\varphi}(0,2 r)} v d \mu_{\varphi}
$$

follows with

$$
B_{2}:=\frac{n\left(C_{n} \varepsilon+2\right) \tilde{C}}{\varepsilon\left(1-\varepsilon C_{n}\right)}
$$

Now, fix $x \in \mathbb{R}^{n}$ and consider a general section $S_{\varphi}(x, r)$. Define

$$
\psi(y):=\varphi(x-y)-\varphi(x)+\langle\nabla \varphi(x), y\rangle .
$$

Then $\mu_{\psi}$ verifies the (DC)-doubling property with the same constants as $\mu_{\varphi}$ does (uniformly in $x$ ), also $\nabla \psi(0)=0, \psi(0)=0$ and

$$
S_{\psi}(0, r)=x-S_{\varphi}(x, r) .
$$

Thus, $\mu_{\psi}\left(S_{\psi}(0, r)\right)=\mu_{\varphi}\left(S_{\varphi}(x, r)\right)$ and if we apply Eq. 5.15 to $\mathcal{L}_{\psi}$ on the open set $x-\Omega$, we obtain the general case just by changing variables $x-y \mapsto y$.

\section{A Mean-Value Inequality for Non-negative Solutions}

A classical result due to F.W. Gehring asserts that weak reverse Hölder inequalities (based on the usual Euclidean balls and Lebesgue measure) enjoy a self-improving property. Such self-improving behavior of the weak reverse Hölder inequality 5.11 (that is, the existence of $q>n /(n-1)$ such that Eq. 5.11 holds with $q$ instead of $n /(n-1)$, with a different constant, but depending on the same parameters) can also be achieved in the context of $\left(\mathbb{R}^{n}, \mu_{\varphi}, \rho_{\varphi}\right)$. Indeed, the underlying structure of space of homogeneous type allows to adapt the higher integrability techniques with doubling measures developed by J. Kinnunen in [17]. However, we will take a different path to actually obtain inequality 1.2 , which represents the extreme case $q=\infty$. In order to do that, we next describe equivalent forms of Eq. 5.11. 
Definition 10 For a section $S_{\varphi}(x, t)$ and a non-negative function $w$ define

$$
\operatorname{aver}\left(w, S_{\varphi}(x, t)\right):=\frac{1}{\mu_{\varphi}\left(S_{\varphi}(x, t)\right)} \int_{S_{\varphi}(x, t)} w(y) d \mu_{\varphi}(y),
$$

and, for a Borel set $E \subset \mathbb{R}^{n}$,

$$
w(E):=\int_{E} w(y) d \mu_{\varphi}(y)
$$

For $R>0$, set

$$
E\left(R, S_{\varphi}(x, t), w\right):=\left\{y \in S_{\varphi}(x, t): w(y) \geq R \operatorname{aver}\left(w, S_{\varphi}(x, 2 t)\right)\right\} .
$$

Given a domain $\Omega \subset \mathbb{R}^{n}$ and $p>1$, we say that a weight $w$ satisfies a weak p-reverse Hölder inequality in $\Omega$ with respect to the structure $\left(\mathbb{R}^{n}, \mu_{\varphi}, \rho_{\varphi}\right)$, and write $w \in W R H_{p}\left(\Omega, \mu_{\varphi}, \rho_{\varphi}\right)$, if there exists a constant $C>0$ such that

$$
\left(\frac{1}{\mu_{\varphi}\left(S_{\varphi}(x, r)\right)} \int_{S_{\varphi}(x, r)} w^{p} d \mu_{\varphi}\right)^{\frac{1}{p}} \leq \frac{C}{\mu_{\varphi}\left(S_{\varphi}(x, 2 r)\right)} \int_{S_{\varphi}(x, 2 r)} w d \mu_{\varphi},
$$

for every $S_{\varphi}(x, r)$ with $S_{\varphi}(x, 2 r) \subset \subset \Omega$.

The following lemma is essentially due to S. Buckley in [3] and E. Sawyer in [19]. We omit its proof since it follows as in Lemma 2.1 in [3] (the proof works in the general context of spaces of homogeneous type)

Lemma 11 Let $p>1$ and $\Omega \subset \mathbb{R}^{n}$ a domain. The following conditions on a weight $w$ are equivalent

(i) $w \in W R H_{p}\left(\Omega, \mu_{\varphi}, \rho_{\varphi}\right)$,

(ii) there exist $\varepsilon>1 / p^{\prime}, C>0$ such that for all Borel subsets $E \subset S_{\varphi}(x, t)$

$$
\frac{w(E)}{w\left(S_{\varphi}(x, 2 t)\right)} \leq C\left(\frac{\mu_{\varphi}(E)}{\mu_{\varphi}\left(S_{\varphi}(x, t)\right)}\right)^{\varepsilon},
$$

for all sections $S_{\varphi}(x, t)$ with $S_{\varphi}(x, 2 t) \subset \subset \Omega$;

(iii) there exists $q>p-1, C>0$ such that

$$
\frac{w\left(E\left(R, S_{\varphi}(x, t), w\right)\right)}{w\left(S_{\varphi}(x, 2 t)\right)} \leq C R^{-q}
$$

for all sections $S_{\varphi}(x, t)$ with $S_{\varphi}(x, 2 t) \subset \subset \Omega$ and all $R>1$;

(iv) there exist $q>p, C>0$ such that

$$
\frac{\mu_{\varphi}\left(E\left(R, S_{\varphi}(x, t), w\right)\right)}{\mu_{\varphi}\left(S_{\varphi}(x, t)\right)} \leq C R^{-q},
$$

for all sections $S_{\varphi}(x, t)$ with $S_{\varphi}(x, 2 t) \subset \subset \Omega$ and all $R>1$.

The statements are quantitative in the sense that the constants involved in each property depend only on each other and not on $w$.

We will also make use of the following critical density result for solutions to the linearized Monge-Ampère equation due to L. Caffarelli and C. Gutiérrez, 
Theorem 12 ([7, Theorem 1]) There are constants $M_{0}>1$ and $0<\varepsilon_{0}<1$, depending only on the (DC)-doubling constants of $\mu_{\varphi}$ and dimension $n$, such that for any section $S_{\varphi}(y, t)$ and any $v$ non-negative solution of $\mathcal{L}_{\varphi} v=0$ in $S_{\varphi}(y, t)$ such that

$$
\inf _{S_{\varphi}(y, t / 2)} v \leq 1
$$

we have that

$$
\mu_{\varphi}\left(\left\{x \in S_{\varphi}(y, t): v(x)>M_{0}\right\}\right) \leq\left(1-\varepsilon_{0}\right) \mu\left(S_{\varphi}(y, t)\right) .
$$

We are now in position to prove a rate of decay for the distribution functions of non-negative super-solutions in terms of their averages (instead of their infima as in Theorem 12). Namely,

Proposition 13 There exist constants $B_{3}>1$ and $q>\frac{n}{n-1}$, depending only on the $(D C)$-doubling constants and dimension, such that if $v$ is a non-negative super-solution to $\mathcal{L}_{\varphi}(v)=0$ in $\Omega$ and if $S_{\varphi}(y, 2 t) \subset \subset \Omega$ with

$$
\frac{1}{\mu_{\varphi}\left(S_{\varphi}(y, t)\right)} \int_{S_{\varphi}(y, t)} v d \mu_{\varphi} \leq 1,
$$

then

$$
\mu_{\varphi}\left(\left\{x \in S_{\varphi}(y, t / 2): v(x) \geq R\right\}\right) \leq B_{3} R^{-q} \mu_{\varphi}\left(S_{\varphi}(y, t / 2)\right), \quad R>1 .
$$

Proof By Theorem 9 we have $v \in W R H_{n /(n-1)}\left(\Omega, \mu_{\varphi}, \rho_{\varphi}\right)$. Given $R>1$, Lemma 11, part (iv), allows to write

$$
\begin{aligned}
\mu_{\varphi} & \left(\left\{x \in S_{\varphi}(y, t / 2): v(x) \geq R\right\}\right) \\
& \leq \mu_{\varphi}\left(\left\{x \in S_{\varphi}(y, t / 2): v(x) \geq R \operatorname{aver}\left(v, S_{\varphi}(y, t)\right)\right\}\right) \\
& =\mu_{\varphi}\left(E\left(R, S_{\varphi}(y, t / 2), v\right) \leq B_{3} R^{-q} \mu_{\varphi}\left(S_{\varphi}(y, t / 2) .\right.\right.
\end{aligned}
$$

Remark 14 There exists a constant $p>0$, depending only on the (DC)-doubling constants for $\mu_{\varphi}$ and dimension $n$, such that whenever $S_{\varphi}(z, 1)$ is a normalized section, $0<s_{1}<s_{2}<1$, and $x \in S_{\varphi}\left(z, s_{1}\right)$, then

$$
S_{\varphi}(x, \rho) \subset S_{\varphi}\left(z, s_{2}\right),
$$

for all $\rho<\left(s_{2}-s_{1}\right)^{p}$ (see [5] or Theorem 3.3.10 in [15]). Also, there exist constants $B_{4}>0$ and $d>0$, depending only on the (DC)-doubling constants for $\mu_{\varphi}$ and dimension $n$, such that $x_{0} \in S_{\varphi}(z, t)$ implies

$$
\mu_{\varphi}\left(S_{\varphi}\left(x_{0}, \rho\right)\right) \geq \frac{1}{B_{4}}\left(\frac{\rho}{t}\right)^{d} \mu_{\varphi}\left(S_{\varphi}(z, t)\right),
$$

for all $\rho<t$. This follows from the doubling condition, see the remark after Lemma 4.1 in [7]. 
Our next result plays the role of Lemma 4.1 in [7], where infima, instead of averages, are treated. For the rest of this section, our techniques will heavily depend on those in [7] and we provide full details for the reader's convenience.

Lemma 15 Assume that $\mu_{\varphi}$ verifies the (DC)-doubling property. Let $M_{0}>1$ and $\varepsilon_{0}>$ 0 be as in Theorem 12 and let $q>n /(n-1)$ and $B_{3}>1$ be as in Proposition 13 (where all of these constants depend only on the (DC)-doubling constants for $\left.\mu_{\varphi}\right)$. Let $u$ be a non-negative solution to $\mathcal{L}_{\varphi}(u)=0$ in $\Omega \subset \mathbb{R}^{n}$. Set

$$
v=\frac{2 M_{0}}{2 M_{0}-1}>1 \text {, }
$$

and suppose that for some section $S_{\varphi}(z, t) \subset S_{\varphi}(z, 2 t) \subset \subset \Omega$ we have

$$
\frac{1}{\mu_{\varphi}\left(S_{\varphi}(z, t)\right)} \int_{S_{\varphi}(z, t)} u(x) d \mu_{\varphi}(x) \leq 1,
$$

and there exist $x_{0} \in S_{\varphi}(z, t / 4)$ and $j \in \mathbb{N}$ verifying

$$
u\left(x_{0}\right) \geq v^{j-1} M_{0},
$$

and

$$
\mu_{\varphi}\left(S_{\varphi}\left(x_{0}, \rho\right)\right) \geq \frac{B_{3}}{\varepsilon_{0}}\left(\frac{v^{j} M_{0}}{2}\right)^{-q} \mu_{\varphi}\left(S_{\varphi}(z, t)\right),
$$

for some $\rho<(t / 4)^{p}$. Then,

$$
\sup _{S_{\varphi}\left(x_{0}, \rho\right)} u \leq v^{j} M_{0}
$$

Proof After an affine change of variables, we can assume $t=1$ and $S_{\varphi}(z, 1)$ normalized (see [7], pp.448-449). As in the proof of Lemma 4.1 in [7], by contradiction we assume $\sup _{S_{\varphi}\left(x_{0}, \rho\right)} u>v^{j} M_{0}$.

Since $x_{0} \in S_{\varphi}(z, 1 / 4)$, by Remark 14 (with $s_{1}=1 / 4$ and $s_{2}=1 / 2$ ), we have $S_{\varphi}\left(x_{0}, \rho\right) \subset S_{\varphi}(z, 1 / 2)$, because $\rho<(1 / 4)^{p}$. For $x \in S_{\varphi}\left(x_{0}, \rho\right)$, define

$$
w(x)=\frac{v^{j} M_{0}-u(x)}{v^{j-1}(v-1) M_{0}} .
$$

It follows that $\mathcal{L}_{\varphi}(w)=0$ and $w \geq 0$ in $S_{\varphi}\left(x_{0}, \rho\right)$, and

$$
w\left(x_{0}\right)=\frac{v^{j} M_{0}-u\left(x_{0}\right)}{v^{j-1}(v-1) M_{0}} \leq \frac{v^{j} M_{0}-v^{j-1} M_{0}}{v^{j-1}(v-1) M_{0}}=1 .
$$

Consequently,

$$
\inf _{S_{\varphi}\left(x_{0}, \rho / 2\right)} w \leq 1
$$

and Theorem 12 yields

$$
\mu_{\varphi}\left(\left\{x \in S_{\varphi}\left(x_{0}, \rho\right): w(x)>M_{0}\right\}\right) \leq\left(1-\varepsilon_{0}\right) \mu_{\varphi}\left(S_{\varphi}\left(x_{0}, \rho\right)\right) .
$$

Next, set

$$
A_{1}=\left\{x \in S_{\varphi}(z, 1 / 2): u(x) \geq v^{j} M_{0} / 2\right\}
$$


and

$$
A_{2}=\left\{x \in S_{\varphi}\left(x_{0}, \rho\right): w(x)>M_{0}\right\}
$$

to obtain

$$
S_{\varphi}\left(x_{0}, \rho\right) \subset A_{1} \cup A_{2} .
$$

Indeed, to prove Eq. 6.23, take $x_{1} \in S_{\varphi}\left(x_{0}, \rho\right) \backslash A_{1}$. Then, $x_{1} \in S_{\varphi}\left(x_{0}, \rho\right)$ and $u\left(x_{1}\right)<$ $v^{j} M_{0} / 2$. Thus,

$$
w\left(x_{1}\right)=\frac{v^{j} M_{0}-u\left(x_{1}\right)}{v^{j-1}(v-1) M_{0}}>\frac{v^{j} M_{0}-\frac{v^{j} M_{0}}{2}}{v^{j-1}(v-1) M_{0}}=\frac{v}{2(v-1)}=M_{0} .
$$

From inequality 6.22, inclusion 6.23, and Proposition 13,

$$
\begin{aligned}
\mu_{\varphi}\left(S_{\varphi}\left(x_{0}, \rho\right)\right) & \leq \mu_{\varphi}\left(A_{1}\right)+\mu_{\varphi}\left(A_{2}\right) \\
& \leq B_{3}\left(\frac{\nu^{j} M_{0}}{2}\right)^{-q} \mu_{\varphi}\left(S_{\varphi}(z, 1)\right)+\left(1-\varepsilon_{0}\right) \mu_{\varphi}\left(S_{\varphi}\left(x_{0}, \rho\right)\right),
\end{aligned}
$$

and then

$$
\mu_{\varphi}\left(S_{\varphi}\left(x_{0}, \rho\right)\right) \leq \frac{B_{3}}{\varepsilon_{0}}\left(\frac{v^{j} M_{0}}{2}\right)^{-q} \mu_{\varphi}\left(S_{\varphi}(z, 1)\right),
$$

contradicting Eq. 6.21.

Remark 16 Notice that, with the notation of Remark 14 and Lemma 15, if we assume

$$
\left(\frac{\rho}{t}\right)^{d} \geq \frac{B_{3} B_{4}}{\varepsilon_{0}}\left(\frac{v^{j} M_{0}}{2}\right)^{-q},
$$

then, by Eq. 6.19, condition 6.21 in Lemma 15 is verified.

Proof of Theorem 3 Let $B_{4}>0, d>0$ and $p>0$ be as in Remark 14. Let $B_{3}>0$ and $q>n /(n-1)$ be as in Proposition 13, and let $M_{0}>1$ and $\varepsilon_{0}>0$ be as in Theorem 12. All these constants depending only on the (DC)-doubling property of $\mu_{\varphi}$. As in the proof of Theorem 5 in [7], set

$$
v=\frac{2 M_{0}}{2 M_{0}-1}>1,
$$

and for each $j \in \mathbb{N}$, define a decreasing sequence $\rho_{j}$ (with limit 0 ) by

$$
\rho_{j}=\left(\frac{B_{3} B_{4}}{\varepsilon_{0}}\right)^{1 / d}\left(\frac{v^{j} M_{0}}{q}\right)^{-q / d} .
$$

Take $S_{\varphi}(z, t)$ and suppose

$$
\frac{1}{\mu_{\varphi}\left(S_{\varphi}(z, t)\right)} \int_{S_{\varphi}(z, t)} u(y) d \mu_{\varphi}(y)=1 .
$$


Again, after an affine change of variables, we can assume $t=1$ and $S_{\varphi}(z, 1)$ normalized. Consider $m \in \mathbb{N}$, depending only on the (DC)-property constants, such that

$$
\sum_{j \geq m} \rho_{j}^{1 / p}<\frac{1}{8}
$$

In particular, we have $\rho_{j}^{1 / p}<1 / 8$ for all $j \geq m$. Our goal is to prove

$$
\sup _{S_{\varphi}(z, 1 / 8)} u \leq v^{m-1} M_{0}
$$

By contradiction, suppose Eq. 6.24 does not hold. Then, there exists $x_{m}$ in $S_{\varphi}(z, 1 / 8)$ with $u\left(x_{m}\right)>v^{m-1} M_{0}$. Since $x_{m} \in S_{\varphi}(z, 1 / 8) \subset S_{\varphi}(z, 1)$ and by the definition of $\rho_{m}$ and Remark 16, we get

$$
\mu_{\varphi}\left(S_{\varphi}\left(x_{m}, \rho_{m}\right)\right) \geq \frac{B_{3}}{\varepsilon_{0}}\left(\frac{\nu^{m} M_{0}}{2}\right)^{-q} \mu_{\varphi}\left(S_{\varphi}(z, 1)\right) .
$$

By Proposition 13, it follows that

$$
\sup _{S_{\varphi}\left(x_{m}, \rho_{m}\right)} u>v^{m} M_{0}
$$

Hence, there exists $x_{m+1} \in S_{\varphi}\left(x_{m}, \rho_{m}\right)$ such that $u\left(x_{m+1}\right)>v^{m} M_{0}$. In addition, by Eq. 6.18, with $s_{1}=1 / 8$ and $s_{2}=\rho^{1 / p}+1 / 8$, we obtain

$$
S_{\varphi}\left(x_{m}, \rho_{m}\right) \subset S_{\varphi}\left(z, \rho_{m}^{1 / p}+1 / 8\right) \subset S_{\varphi}(z, 1 / 4) .
$$

Hence, $x_{m+1} \in S_{\varphi}\left(z, \rho_{m}^{1 / p}+1 / 8\right) \subset S_{\varphi}(z, 1 / 4)$. Also,

$$
\mu_{\varphi}\left(S_{\varphi}\left(x_{m+1}, \rho_{m}\right)\right) \geq \frac{B_{3}}{\varepsilon_{0}}\left(\frac{\nu^{m+1} M_{0}}{2}\right)^{-q} \mu_{\varphi}\left(S_{\varphi}(z, 1)\right),
$$

again by the choice of $\rho_{m}$ and Remark 16. By Proposition 13, we must have

$$
\sup _{S_{\varphi}\left(x_{m+1}, \rho_{m+1}\right)} u>v^{m+1} M_{0}
$$

Notice that, by Eq. 6.18, with $s_{1}=1 / 8+\rho_{m}^{p}$ and $s_{2}=1 / 8+\rho_{m}^{p}+\rho_{m+1}^{p}$, it follows that

$$
S_{\varphi}\left(x_{m+1}, \rho_{m+1}^{p}\right) \subset S_{\varphi}\left(z, 1 / 8+\rho_{m}^{p}+\rho_{m+1}^{p}\right) \subset S_{\varphi}(z, 1 / 4) .
$$

Iterations of this argument provide a sequence $\left\{x_{j}\right\}_{j=m}^{\infty}$ such that

$$
u\left(x_{j}\right) \geq v^{j-1} M_{0}
$$

and

$$
x_{j} \in S_{\varphi}\left(z, 1 / 8+\rho_{m}^{1 / p}+\cdots+\rho_{j-1}^{1 / p}\right) \subset S_{\varphi}(z, 1 / 4),
$$


which implies

$$
\sup _{S_{\varphi}(z, 1 / 4)} u=\infty
$$

contradicting the continuity of the solution $u$ (see Remark 4). Therefore, Eq. 6.24 holds true and Theorem 3 follows.

\section{An Application to Affine Maximal Hypersurfaces}

Let $\Omega \subset \mathbb{R}^{n}$ be a domain and $\varphi \in C^{4}(\Omega)$. We follow [21] and say that $\varphi$ is affine maximal in $\Omega$ if $\varphi$ satisfies the following affine maximal surface equation

$$
\left\{\begin{aligned}
\mathcal{L}_{\varphi}(w) & =0, \\
w & =\left(\operatorname{det} D^{2} \varphi\right)^{-\frac{n+1}{n+2}}
\end{aligned}\right.
$$

in $\Omega$. Estimates for the solutions to Eq. 7.25 of the form

$$
\lambda \leq \operatorname{det} D^{2} \varphi(x) \leq \Lambda, \quad x \in \Omega,
$$

for some positive numbers $\lambda$ and $\Lambda$, play a significant role in the recent work of $\mathrm{N}$. Trudinger and X.-J. Wang on the Bernstein and Plateau problems [21-23]. In their estimates, however, the constants $\lambda$ and $\Lambda$ depend heavily on the solutions (and, in particular, on their modulus of convexity) and on the domain $\Omega$, see, for instance, [21], p. 407. In their approach, the fact that the normalization of solutions changes their modulus of convexity (that is, the eccentricity of the resulting sections) prevents from obtaining uniform constants $\lambda$ and $\Lambda$ in Eq. 7.26.

On the other hand, as we mentioned in Section 4, the normalization of solutions does not change their doubling constants in Eq. 1.1. Since the normalization technique has proven to be exceedingly useful in the development of the Monge-Ampère regularity theory, the use of the normalization-invariant condition 1.1 relaxes any hypothesis on uniform moduli of convexity. Along these lines, the following result allows to obtain uniform bounds $\lambda$ and $\Lambda$ for entire solutions verifying the doubling condition 1.1.

Theorem 17 Let $\varphi \in C^{4}\left(\mathbb{R}^{n}\right)$ be a strictly convex solution to the Eq. 7.25 in a domain $\Omega$. If $\mu_{\varphi}=\operatorname{det} D^{2} \varphi$ satisfies the doubling condition 1.1 , then there exist constants $B_{5}>$ 1 and $0<\sigma<1$, depending only on the doubling constants in Eq. 1.1 and dimension, such that

$$
\sup _{S_{\varphi}(x, \sigma r)} \operatorname{det} D^{2} \varphi \leq B_{5} \inf _{S_{\varphi}(x, \sigma r)} \operatorname{det} D^{2} \varphi
$$

for all sections $S(x, r)$ with $S(x, 2 r) \subset \subset \Omega$. In particular, if $\varphi$ satisfies $E q .7 .25$ in all of $\mathbb{R}^{n}$, then there exist positive constants $\lambda$ and $\Lambda$, depending only on the doubling constants in Eq. 1.1 and dimension, such that

$$
\lambda \operatorname{det} D^{2} \varphi(y) \leq \operatorname{det} D^{2} \varphi(x) \leq \Lambda \operatorname{det} D^{2} \varphi(y), \quad x, y \in \mathbb{R}^{n} .
$$


Proof By Theorem 3 applied to the (non-negative) solution $w=\left(\operatorname{det} D^{2} \varphi\right)^{-\frac{n+1}{n+2}}$ in Eq. 7.25 and using Jensen's inequality, we obtain

$$
\begin{aligned}
\sup _{S_{\varphi}(x, r / 8)} \mu_{\varphi}^{-\frac{n+1}{n+2}} & \leq \frac{B_{1}}{\mu_{\varphi}\left(S_{\varphi}(x, r)\right)} \int_{S_{\varphi}(x, r)} \mu_{\varphi}^{-\frac{n+1}{n+2}} \mu_{\varphi} \\
& =\frac{B_{1}}{\mu_{\varphi}\left(S_{\varphi}(x, r)\right)} \int_{S_{\varphi}(x, r)} \mu_{\varphi}^{\frac{1}{n+2}} \\
& \leq \frac{B_{1}\left|S_{\varphi}(x, r)\right|}{\mu_{\varphi}\left(S_{\varphi}(x, r)\right)}\left(\frac{1}{\left|S_{\varphi}(x, r)\right|} \int_{S_{\varphi}(x, r)} \mu_{\varphi}\right)^{\frac{1}{n+2}} \\
& =B_{1}\left(\frac{\left|S_{\varphi}(x, r)\right|}{\mu_{\varphi}\left(S_{\varphi}(x, r)\right)}\right)^{\frac{n+1}{n+2}},
\end{aligned}
$$

for every section $S_{\varphi}(x, r)$ with $S_{\varphi}(x, 2 r) \subset \subset \Omega$. Hence,

$$
\frac{\mu_{\varphi}\left(S_{\varphi}(x, r)\right)}{\left|S_{\varphi}(x, r)\right|} \leq B_{1}^{\frac{n+2}{n+1}} \inf _{S_{\varphi}(x, r / 8)} \mu_{\varphi} .
$$

On the other hand, the Legendre transform $\varphi^{*}$ defined for $y \in \Omega^{*}:=\nabla \varphi(\Omega)$ by

$$
\varphi^{*}(y)=\sup _{x \in \Omega}(\langle x, y\rangle-\varphi(x))
$$

turns out to be strictly convex and $C^{4}$ in $\Omega^{*}$ (Theorem 26.5 in [18]). By Theorem 12 in [13] we have that $\mu_{\varphi^{*}}=\operatorname{det} D^{2} \varphi^{*}$ verifies the doubling condition 1.1 with constants depending only on the ones for $\mu_{\varphi}$ (recall that, by Theorem 5, the doubling condition 1.1 and the engulfing property are equivalent). Moreover, as in [21], p. 407, $\varphi^{*}$ satisfies the following equation

$$
\left\{\begin{aligned}
\mathcal{L}_{\varphi^{*}}\left(w^{*}\right) & =0 \\
w^{*} & =\left(\operatorname{det} D^{2} \varphi^{*}\right)^{-\frac{1}{n+2}}
\end{aligned}\right.
$$

in $\Omega^{*}$. Now, by Theorem 3 applied to the function $\varphi^{*}$ and the solution $w^{*}=$ $\left(\operatorname{det} D^{2} \varphi^{*}\right)^{-\frac{1}{n+2}}$ we obtain

$$
\sup _{S_{\varphi^{*}}(y, r / 8)} \mu_{\varphi^{*}}^{-\frac{1}{n+2}} \leq \frac{B_{1}^{*}}{\mu_{\varphi^{*}}\left(S_{\varphi^{*}}(y, r)\right)} \int_{S_{\varphi^{*}}(y, r)} \mu_{\varphi^{*}}^{-\frac{1}{n+2}} \mu_{\varphi^{*}},
$$

for all sections $S_{\varphi^{*}}(y, r)$ with $S_{\varphi^{*}}(y, 2 r) \subset \subset \Omega^{*}$, where the constant $B_{1}^{*}$ depends only on the doubling constants for $\mu_{\varphi^{*}}$ (and hence on those of $\mu_{\varphi}$ ) and dimension $n$. Reasoning as before, Eq. 7.31 implies

$$
\frac{\mu_{\varphi^{*}}\left(S_{\varphi^{*}}(y, r)\right)}{\left|S_{\varphi^{*}}(y, r)\right|} \leq\left(B_{1}^{*}\right)^{n+2} \inf _{S_{\varphi^{*}}(y, r / 8)} \mu_{\varphi^{*}}
$$

Finally, we will relate inequalities 7.29 and 7.32 by using the change of variables $y=$ $\nabla \varphi(x)$. By Theorem 12 and Corollary 13 in [13], $\nabla \varphi: \mathbb{R}^{n} \rightarrow \mathbb{R}^{n}$ is a homeomorphism with inverse $\nabla \varphi^{*}$ and there exists a number $K$, depending only on the doubling constants for $\mu_{\varphi}$ in Eq. 1.1 and dimension $n$, such that

$$
\nabla \varphi\left(S_{\varphi}(x, t / K)\right) \subset S_{\varphi^{*}}(\nabla \varphi(x), t) \subset \nabla \varphi\left(S_{\varphi}(x, K t)\right), \quad x \in \mathbb{R}^{n}, t>0 .
$$


(The exact dependence of $K$ on the doubling constants can be found in Section 7 of [13]). The inclusions in Eq. 7.33 imply, by taking Lebesgue measure, that

$$
\begin{aligned}
\mu_{\varphi}\left(S_{\varphi}(x, t / K)\right) & =\left|\nabla \varphi\left(S_{\varphi}(x, t / K)\right)\right| \leq\left|S_{\varphi^{*}}(\nabla \varphi(x), t)\right| \\
& \leq\left|\nabla \varphi\left(S_{\varphi}(x, K t)\right)\right|=\mu_{\varphi}\left(S_{\varphi}(x, K t)\right), \quad x \in \mathbb{R}^{n}, t>0 .
\end{aligned}
$$

Similarly,

$$
\left|S_{\varphi}(x, t / K)\right| \leq \mu_{\varphi^{*}}\left(S_{\varphi^{*}}(\nabla \varphi(x), t)\right)=\left|\nabla \varphi^{*}\left(S_{\varphi^{*}}(\nabla \varphi(x), t)\right)\right| \leq\left|S_{\varphi}(x, K t)\right|,
$$

for all $x \in \mathbb{R}^{n}$ and $t>0$. Also,

$$
\begin{aligned}
\inf _{S_{\varphi^{*}}(y, r / 8)} \mu_{\varphi^{*}} & =\inf _{S_{\varphi^{*}}(y, r / 8)} \operatorname{det} D^{2} \varphi^{*}=\inf _{z \in S_{\varphi^{*}}(y, r / 8)}\left(\frac{1}{\operatorname{det} D^{2} \varphi\left(\nabla \varphi^{*}(z)\right)}\right) \\
& =\left(\sup _{\nabla \varphi^{*}\left(S_{\left.\varphi^{*}(y, r / 8)\right)} \operatorname{det} D^{2} \varphi\right)^{-1} \leq\left(\sup _{S_{\varphi}(y, r /(8 K))} \operatorname{det} D^{2} \varphi\right)^{-1} .}\right.
\end{aligned}
$$

Consequently, Eq. 7.27 follows (with $\sigma=1 /(8 K)$ ) by the doubling properties of $\mu_{\varphi}$ and $\mu_{\varphi^{*}}$, and Eq. 3.7. Clearly, Eq. 7.28 follows from Eq. 7.27 in the case $\Omega=\mathbb{R}^{n}$ with $\Lambda=\lambda^{-1}=B_{5}$.

Acknowledgement The authors would like to thank the anonimous referee for his/her helpful comments.

\section{References}

1. Aimar, H., Forzani, L., Toledano, R.: Balls and quasi-metrics: a space of homogeneous type modeling the real analysis related to the Monge-Ampère equation. J. Fourier Anal. Appl. 4, 377-381 (1998)

2. Brenier, Y.: Dcomposition polaire et rarrangement monotone des champs de vecteurs. (French) [Polar decomposition and increasing rearrangement of vector fields]. C. R. Acad. Sci. Paris Sér. I Math. 305(19), 805-808 (1987)

3. Buckley, S.: Pointwise multipliers for reverse Hölder spaces II. Proc. Roy. Irish Acad. Sect. A 95, 193-204 (1995)

4. Caffarelli, L.: Some regularity properties of solutions of Monge-Ampère equation. Comm. Pure Appl. Math. 44, 965-969 (1991)

5. Caffarelli, L.: Boundary regularity of maps with convex potentials. Comm. Pure Appl. Math. 45(9), 1141-1151 (1992)

6. Caffarelli, L., Gutiérrez, C.: Real analysis related to the Monge-Ampère equation. Trans. Amer. Math. Soc. 348, 1075-1092 (1996)

7. Caffarelli, L., Gutiérrez: C.: Properties of the solutions of the linearized Monge-Ampère equation. Amer. J. Math. 119(2) (1997), 423-465.

8. Caffarelli, L., Silvestre, L.: An extension problem related to the fractional Laplacian. Comm. P.D.E. 32(8), 1245-1260 (2007)

9. Cruz-Uribe, D.: The minimal operator and the geometric maximal operator in $\mathbb{R}^{n}$. Studia Math. 144(1), 1-37 (2001)

10. Cullen, M., Norbury, J., Purser, R.: Generalised Lagrangian solutions for atmospheric and oceanic flows. SIAM J. Appl. Math. 51(1), 20-31 (1991)

11. Fabes, E., Stroock, W.: The $L^{p}$-integrability of Green's functions and fundamental solutions for elliptic and parabolic equations. Duke Math. J. 51(4), 997-1016 (1984)

12. Forzani, L., Maldonado, D.: On geometric characterizations for Monge-Ampère doubling measures. J. Math. Anal. Appl. 275(2), 721-732 (2002)

13. Forzani, L., Maldonado, D.: Properties of the solutions to the Monge-Ampère equation. Nonlinear Anal. 57(5-6), 815-829 (2004) 
14. Gilbarg, D., Trudinger, M.: Elliptic Partial Differential Equations of Second Order. Springer, New York (2001)

15. Gutiérrez, C.: The Monge-Ampère Equation. Progress in Nonlinear Differential Equations and Their Applications, vol. 44. Birkhäuser, Boston (2001)

16. Gutiérrez C., Huang, Q.: Geometric properties of the sections of solutions to the Monge-Ampère equation. Trans. Amer. Math. Soc. 352(9), 4381-4396 (2000)

17. Kinnunen, J.: Higher integrability with weights. Ann. Acad. Sci. Fenn. Ser. A I Math. 19, 355-366 (1994)

18. Rockafellar, R.T..: Convex Analysis. Princeton University Press, Princeton (1970)

19. Sawyer, E.: Two weight inequalities for certain maximal and integral operators. In: Lecture Notes in Mathematics, vol. 908. Springer, New York (1982)

20. Stein, E.M.: Harmonic Analysis: Real Variable Methods, Orthogonality, and Oscillatory Integrals. Princeton University Press, Princeton NJ (1993)

21. Trudinger, N., Wang, X.-J.: The Bernstein problem for affine maximal hypersurfaces. Invent. Math. 140(2), 399-422 (2000)

22. Trudinger, N., Wang, X.-J: Affine complete locally convex hypersurfaces. Invent. Math. 150(1), 45-60 (2002)

23. Trudinger, N., Wang, X.-J: The affine Plateau problem. J. Am. Math. Soc. 18(2), 253-289 (2005)

24. Wik, I.: On Muckenhoupt's classes of weight functions. Stud. Math. 94(3), 245-255 (1989) 